\title{
Exact Travelling Wave Solutions to the $(3+1)$-Dimensional Kadomtsev-Petviashvili Equation
}

\author{
Y.-Z. PENG ${ }^{a *}$ AND E.V. KRISHNAN ${ }^{b}$ \\ ${ }^{a}$ Department of Mathematics, Huazhong University of Science \\ and Technology, Wuhan 430074, P.R. China \\ ${ }^{b}$ Department of Mathematics and Statistics, Sultan Qaboos University \\ P.O. Box 36, Al-Khod 123, Muscat, Sultanate of Oman
}

(Received December 29, 2004)

\begin{abstract}
Exact travelling wave solutions in terms of the Jacobi elliptic functions are obtained to the $(3+1)$-dimensional Kadomtsev-Petviashvili equation by means of the extended mapping method. Limit cases are studied, and new solitary wave solutions and trigonometric periodic wave solutions are got. The method is applicable to a large variety of nonlinear partial differential equations.
\end{abstract}

PACS numbers: 02.30.Jr

\section{Introduction}

Travelling waves, whether their solution expressions are in explicit or implicit forms, are very interesting from the point of view of applications. These types of waves will not change their shapes during propagation and are thus easy to detect. Of particular interest are three types of travelling waves: the solitary waves, which are localized travelling waves, asymptotically zero at large distances, the periodic waves, and the kink waves, which rise or descend from one asymptotic state to another. Recently, a unified algebraic method, called the mapping method [1-4], is proposed to obtain exact travelling wave solutions for a large variety of nonlinear partial differential equations (PDEs). This method includes several direct methods as special cases, such as tanh-function method, sech-function method, and Jacobi elliptic function method. Above all, by means of this method, the solitary wave, the periodic wave, and the kink wave (or the shock wave) solutions can, if they exist, be obtained simultaneously to the equation in question without extra

*e-mail: yanzepeng@163.com 
efforts. The basic idea of the method is as follows. For a given nonlinear evolution equation, say, in four variables

$$
N\left(u, u_{t}, u_{x}, u_{y}, u_{z}, u_{x x}, \cdots\right)=0 .
$$

We seek for its travelling wave solution of the form

$$
u(x, t) \equiv u(\xi), \quad \xi=k_{1} x+k_{2} y+k_{3} z-\omega t .
$$

Substituting Eq. (2) into Eq. (1) yields an ordinary differential equation of $u(\xi)$. Then $u(\xi)$ is expanded into a polynomial in $f(\xi)$

$$
u(\xi)=\sum_{i=0}^{n} A_{i} f^{i},
$$

where $A_{i}$ are constants to be determined, $n$ is fixed by balancing the linear term of the highest order with nonlinear term in Eq. (1), and $f$ satisfies the following equation (the first kind of elliptic equation)

$$
f^{\prime \prime}=p f+q f^{3}, \quad f^{\prime 2}=p f^{2}+\frac{1}{2} q f^{4}+r,
$$

where $p, q$, and $r$ are constants to be determined. After Eq. (3) with Eq. (4) is substituted into the ordinary differential equation, the coefficients $A_{i}, k_{i}, \omega, p, q$, and $r$ may be determined. If any of the parameters is left unspecified, it is regarded as being arbitrary for the solution to Eq. (1). Thus Eq. (3) establishes an algebraic mapping relation between the solution to Eq. (4) and that of Eq. (1), and this method is called the mapping method. By virtue of it, a series of Jacobi elliptic wave solutions to some physically important nonlinear PDEs was obtained [1-4]. The Jacobi elliptic functions $\operatorname{sn} \xi=\operatorname{sn}(\xi \mid m), \operatorname{cn} \xi=\mathrm{cn}(\xi \mid m)$, and $\operatorname{dn} \xi=\operatorname{dn}(\xi \mid m)$, where $m(0<m<1)$ is the modulus of the elliptic function, are double periodic and possess properties of trigonometric functions, namely, $\operatorname{sn}^{2} \xi+\mathrm{cn}^{2} \xi=1, \operatorname{dn}^{2} \xi+m^{2} \operatorname{sn}^{2} \xi=1,(\operatorname{sn} \xi)^{\prime}=\operatorname{cn} \xi \operatorname{dn} \xi, \quad(\operatorname{cn} \xi)^{\prime}=-\operatorname{sn} \xi \operatorname{dn} \xi$, $(\operatorname{dn} \xi)^{\prime}=-m^{2} \operatorname{sn} \xi \operatorname{cn} \xi$. When $m \rightarrow 0$, the Jacobi elliptic functions degenerate to the triangular functions, i.e., $\operatorname{sn} \xi \rightarrow \sin \xi, \operatorname{cn} \xi \rightarrow \cos \xi, \operatorname{dn} \xi \rightarrow 1$. When $m \rightarrow 1$, the Jacobi elliptic functions degenerate to the hyperbolic functions, i.e. $\operatorname{sn} \xi \rightarrow \tanh \xi, \operatorname{cn} \xi \rightarrow \operatorname{sech} \xi, \operatorname{dn} \xi \rightarrow \operatorname{sech} \xi$. Detailed explanations about Jacobi elliptic functions can be found in Refs. $[5,6]$. Note that although the other 9 Jacobi elliptic functions are all expressible in terms of $\operatorname{sn} \xi, \mathrm{cn} \xi$, and $\operatorname{dn} \xi$, we must allow $m$ to be outside the range of $0<m<1$ [6]. In this paper, we will introduce a new expansion,

$$
u=A_{0}+\sum_{i=1}^{n} f^{i-1}\left(A_{i} f+B_{i} g\right),
$$

to take the place of Eq. (3), where $f$ and $g$ satisfy Eq. (4) and

$$
g^{\prime \prime}=g\left(c_{1}+c_{2} f^{2}\right), \quad g^{2}=c_{3}+c_{4} f^{2}, \quad f^{\prime} g^{\prime}=f g\left(c_{5}+c_{6} f^{2}\right),
$$


respectively. We call this approach the extended mapping method. When $B_{i}=0$, it reduces to the mapping method. Due to the entrance of the parameters $p, q, r$, and $c_{i}$, Eqs. (4) and (6) have a rich structure of solutions. As $p=-2, q=2, r=1$ and $c_{1}=-1, c_{2}=2, c_{3}=1, c_{4}=-1, c_{5}=-1, c_{6}=1$, for example, the solution reads $f(\xi)=\tanh \xi, g(\xi)=\operatorname{sech} \xi$, and the method is called the twofamily truncation method $[7,8]$. As a simple application of the method, we will use the extended mapping method to obtain abundant travelling wave solutions to the $(3+1)$-dimensional Kadomtsev-Petviashvili (KP) equation.

\section{Exact solutions to the $(3+1)$-dimensional KP equation}

The $(3+1)$-dimensional KP equation

$$
\left(u_{t}+6 u u_{x}+u_{x x x}\right)_{x}-3\left(u_{y y}+u_{z z}\right)=0,
$$

explains wave propagation in the field of plasma physics, fluid dynamics, etc. $[9,10]$. Soliton simulation studies for Eq. (7) have been done by Senatorski et al. [11]. Equation (7) is not integrable by inverse scattering transformation unless $\partial_{z}=0$, but it passes the Painleve property [12]. In what follows, we study the travelling wave solutions to Eq. (7). Substituting $u=u(\xi), \xi=k_{1} x+k_{2} y+k_{3} z-\omega t$ into Eq. (7) and integrating twice, we have

$$
k_{1}^{4} u^{\prime \prime}+3 k_{1}^{2} u^{2}-\left(\omega k_{1}+3 k_{2}^{2}+3 k_{3}^{2}\right) u=C,
$$

where $C$ is the integration constant, and the first integrating constant is taken to zero. According to the method described above, we assume that Eq. (8) has the solution in the form

$$
u=A_{0}+A_{1} f+B_{1} g+A_{2} f^{2}+B_{2} f g,
$$

where $A_{i}$ and $B_{i}$ are constants to be determined, and $f$ and $g$ satisfy Eqs. (4) and (6). Substituting Eq. (9) with Eqs. (4) and (6) into Eq. (8) and equating the coefficients of like powers of $f^{i} g^{j}(j=0,1)$, we find two sets of solutions

$$
\begin{aligned}
& A_{0}=-\frac{4 p k_{1}^{4}-\left(\omega k_{1}+3 k_{2}^{2}+3 k_{3}^{2}\right)}{6 k_{1}^{2}}, \\
& A_{2}=-q k_{1}^{2}, \quad A_{1}=B_{1}=B_{2}=0,
\end{aligned}
$$

and

$$
\begin{aligned}
& A_{0}=-\frac{\left(p+c_{1}+2 c_{5}\right) k_{1}^{4}-\left(\omega k_{1}+3 k_{2}^{2}+3 k_{3}^{2}\right)}{6 k_{1}^{2}}, \\
& A_{2}=-\frac{1}{6}\left(q+c_{2}+2 c_{6}\right) k_{1}^{2}, \quad A_{1}=B_{1}=0, \\
& B_{2}^{2}=\frac{\left(3 p-c_{1}-2 c_{5}\right)\left(q+c_{2}+2 c_{6}\right) k_{1}^{4}}{18 c_{3}} \\
& c_{3}\left(-5 q+c_{2}+2 c_{6}\right)+2 c_{4}\left(3 p-c_{1}-2 c_{5}\right)=0 .
\end{aligned}
$$


So, we obtain the exact solutions of Eq. (7) as follows:

$$
u=-\frac{4 p k_{1}^{4}-\left(\omega k_{1}+3 k_{2}^{2}+3 k_{3}^{2}\right)}{6 k_{1}^{2}}-q k_{1}^{2} f^{2}(\xi)
$$

where $f$ satisfies Eq. (4) and

$$
\begin{aligned}
u= & -\frac{\left(p+c_{1}+2 c_{5}\right) k_{1}^{4}-\left(\omega k_{1}+3 k_{2}^{2}+3 k_{3}^{2}\right)}{6 k_{1}^{2}}-\frac{1}{6}\left(q+c_{2}+2 c_{6}\right) k_{1}^{2} f^{2}(\xi) \\
& \pm \frac{1}{3} k_{1}^{2} \sqrt{\frac{\left(3 p-c_{1}-2 c_{5}\right)\left(q+c_{2}+2 c_{6}\right)}{2 c_{3}}} f(\xi) g(\xi),
\end{aligned}
$$

where $f$ and $g$ satisfy Eqs. (4) and (6) with the constraint among the parameters

$$
c_{3}\left(-5 q+c_{2}+2 c_{6}\right)+2 c_{4}\left(3 p-c_{1}-2 c_{5}\right)=0,
$$

and $\xi=k_{1} x+k_{2} y+k_{3} z-\omega t$. The solution Eq. (12) can also be got by means of the mapping method [1-4], and the discussion about its specific expressions is only a routine thing following Refs. [1-4]. Therefore, as examples, we study only the solution Eq. (13) in what follows.

Case 1. $p=-\left(1+m^{2}\right), q=2 m^{2}, r=1$

Subcase 1.1. $c_{1}=-1, c_{2}=2 m^{2}, c_{3}=1, c_{4}=-1, c_{5}=-1, c_{6}=m^{2}$

Equations (4) and (6) have the solution $f(\xi)=\operatorname{sn} \xi, g(\xi)=\operatorname{cn} \xi$. So we obtain the periodic wave solution of Eq. (7)

$$
\begin{aligned}
u= & \frac{\left(m^{2}+4\right) k_{1}^{4}+\left(\omega k_{1}+3 k_{2}^{2}+3 k_{3}^{2}\right)}{6 k_{1}^{2}}-k_{1}^{2} m^{2} \operatorname{sn}^{2}\left(k_{1} x+k_{2} y+k_{3} z-\omega t\right) \\
& \pm \mathrm{i} k_{1}^{2} m^{2} \operatorname{sn}\left(k_{1} x+k_{2} y+k_{3} z-\omega t\right) \operatorname{cn}\left(k_{1} x+k_{2} y+k_{3} z-\omega t\right) .
\end{aligned}
$$

As $m \rightarrow 1$, from Eq. (14) one has

$$
\begin{aligned}
u= & \frac{5 k_{1}^{4}+\left(\omega k_{1}+3 k_{2}^{2}+3 k_{3}^{2}\right)}{6 k_{1}^{2}}-k_{1}^{2} \tanh ^{2}\left(k_{1} x+k_{2} y+k_{3} z-\omega t\right) \\
& \pm \mathrm{i} k_{1}^{2} \tanh \left(k_{1} x+k_{2} y+k_{3} z-\omega t\right) \operatorname{sech}\left(k_{1} x+k_{2} y+k_{3} z-\omega t\right),
\end{aligned}
$$

a complex line solitary wave solution to Eq. (7).

Subcase 1.2. $c_{1}=-m^{2}, c_{2}=2 m^{2}, c_{3}=1, c_{4}=-m^{2}, c_{5}=-m^{2}, c_{6}=m^{2}$

The solution of Eqs. (4) and (6) is $f(\xi)=\operatorname{sn} \xi, g(\xi)=\operatorname{dn} \xi$. Hence another periodic wave solution to Eq. (7) reads

$$
\begin{aligned}
u= & \frac{\left(4 m^{2}+1\right) k_{1}^{4}+\left(\omega k_{1}+3 k_{2}^{2}+3 k_{3}^{2}\right)}{6 k_{1}^{2}}-k_{1}^{2} m^{2} \operatorname{sn}^{2}\left(k_{1} x+k_{2} y+k_{3} z-\omega t\right) \\
& \pm \mathrm{i} k_{1}^{2} m \operatorname{sn}\left(k_{1} x+k_{2} y+k_{3} z-\omega t\right) \operatorname{dn}\left(k_{1} x+k_{2} y+k_{3} z-\omega t\right) .
\end{aligned}
$$

As $m \rightarrow 1$, Eq. (16) degenerates to Eq. (15). 
Subcase 1.3. $c_{1}=-m^{2}, c_{2}=2 m^{2}, c_{3}=\frac{1}{1-m^{2}}, c_{4}=-\frac{m^{2}}{1-m^{2}}, c_{5}=-m^{2}, c_{6}=m^{2}$

We have $f(\xi)=\operatorname{cd} \xi \equiv \operatorname{cn} \xi / \operatorname{dn} \xi, g(\xi)=\operatorname{nd} \xi \equiv 1 / \operatorname{dn} \xi$. So the periodic wave solution to Eq. (7) is

$$
\begin{aligned}
u= & \frac{\left(4 m^{2}+1\right) k_{1}^{4}+\left(\omega k_{1}+3 k_{2}^{2}+3 k_{3}^{2}\right)}{6 k_{1}^{2}}-k_{1}^{2} m^{2} \mathrm{~cd}^{2}\left(k_{1} x+k_{2} y+k_{3} z-\omega t\right) \\
& \pm \mathrm{i} k_{1}^{2} m \sqrt{1-m^{2}} \mathrm{~cd}\left(k_{1} x+k_{2} y+k_{3} z-\omega t\right) \operatorname{nd}\left(k_{1} x+k_{2} y+k_{3} z-\omega t\right) .
\end{aligned}
$$

Subcase 1.4. $c_{1}=-1, c_{2}=2 m^{2}, c_{3}=\frac{1}{1-m^{2}}, c_{4}=-\frac{1}{1-m^{2}}, c_{5}=-1, c_{6}=m^{2}$

The solution of Eqs. (4) and (6) is $f(\xi)=\operatorname{cd} \xi, g(\xi)=\operatorname{sd} \xi \equiv \operatorname{sn} \xi / \operatorname{dn} \xi$. Thus we get

$$
\begin{aligned}
u= & \frac{\left(m^{2}+4\right) k_{1}^{4}+\left(\omega k_{1}+3 k_{2}^{2}+3 k_{3}^{2}\right)}{6 k_{1}^{2}}-k_{1}^{2} m^{2} \operatorname{cd}^{2}\left(k_{1} x+k_{2} y+k_{3} z-\omega t\right) \\
& \pm \mathrm{i} k_{1}^{2} m^{2} \sqrt{1-m^{2}} \mathrm{~cd}\left(k_{1} x+k_{2} y+k_{3} z-\omega t\right) \\
& \times \operatorname{sd}\left(k_{1} x+k_{2} y+k_{3} z-\omega t\right) .
\end{aligned}
$$$$
\text { Case 2. } p=-\left(1+m^{2}\right), q=2, r=m^{2}
$$

Subcase 2.1. $c_{1}=-m^{2}, c_{2}=2, c_{3}=-1, c_{4}=1, c_{5}=-m^{2}, c_{6}=1$

Equations (4) and (6) have the solution $f(\xi)=\mathrm{ns} \xi \equiv 1 / \operatorname{sn} \xi, g(\xi)=\operatorname{cs} \xi \equiv$ $\operatorname{cn} \xi / \operatorname{sn} \xi$. Therefore, we obtain the periodic wave solution of Eq. (7)

$$
\begin{aligned}
u= & \frac{\left(4 m^{2}+1\right) k_{1}^{4}+\left(\omega k_{1}+3 k_{2}^{2}+3 k_{3}^{2}\right)}{6 k_{1}^{2}}-k_{1}^{2} \mathrm{~ns}^{2}\left(k_{1} x+k_{2} y+k_{3} z-\omega t\right) \\
& \pm k_{1}^{2} \mathrm{~ns}\left(k_{1} x+k_{2} y+k_{3} z-\omega t\right) \operatorname{cs}\left(k_{1} x+k_{2} y+k_{3} z-\omega t\right) .
\end{aligned}
$$

As $m \rightarrow 0$ and $m \rightarrow 1$, Eq. (19) degenerates to

$$
\begin{aligned}
u= & \frac{k_{1}^{4}+\left(\omega k_{1}+3 k_{2}^{2}+3 k_{3}^{2}\right)}{6 k_{1}^{2}}-k_{1}^{2} \csc ^{2}\left(k_{1} x+k_{2} y+k_{3} z-\omega t\right) \\
& \pm k_{1}^{2} \csc \left(k_{1} x+k_{2} y+k_{3} z-\omega t\right) \cot \left(k_{1} x+k_{2} y+k_{3} z-\omega t\right),
\end{aligned}
$$

and

$$
\begin{aligned}
u= & \frac{5 k_{1}^{4}+\left(\omega k_{1}+3 k_{2}^{2}+3 k_{3}^{2}\right)}{6 k_{1}^{2}}-k_{1}^{2} \operatorname{coth}^{2}\left(k_{1} x+k_{2} y+k_{3} z-\omega t\right) \\
& \pm k_{1}^{2} \operatorname{coth}\left(k_{1} x+k_{2} y+k_{3} z-\omega t\right) \operatorname{csch}\left(k_{1} x+k_{2} y+k_{3} z-\omega t\right),
\end{aligned}
$$

respectively.

Subcase 2.2. $c_{1}=-1, c_{2}=2, c_{3}=-m^{2}, c_{4}=1, c_{5}=-1, c_{6}=1$

The solution of Eqs. (4) and (6) reads $f(\xi)=\mathrm{ns} \xi, g(\xi)=\mathrm{ds} \xi \equiv \mathrm{dn} \xi / \mathrm{sn} \xi$. So another periodic wave solution of Eq. (7) is 


$$
\begin{aligned}
u= & \frac{\left(m^{2}+4\right) k_{1}^{4}+\left(\omega k_{1}+3 k_{2}^{2}+3 k_{3}^{2}\right)}{6 k_{1}^{2}}-k_{1}^{2} \mathrm{~ns}^{2}\left(k_{1} x+k_{2} y+k_{3} z-\omega t\right) \\
& \pm k_{1}^{2} \mathrm{~ns}\left(k_{1} x+k_{2} y+k_{3} z-\omega t\right) \mathrm{ds}\left(k_{1} x+k_{2} y+k_{3} z-\omega t\right) .
\end{aligned}
$$

As $m \rightarrow 1$, we obtain Eq. (21) again.

Subcase 2.3. $c_{1}=-1, c_{2}=2, c_{3}=-\frac{m^{2}}{1-m^{2}}, c_{4}=\frac{1}{1-m^{2}}, c_{5}=-1, c_{6}=1$

Equations (4) and (6) have the solution $f(\xi)=\mathrm{dc} \xi, g(\xi)=\mathrm{nc} \xi \equiv 1 / \mathrm{cn} \xi$. So the new periodic wave solution is

$$
\begin{aligned}
u= & \frac{\left(m^{2}+4\right) k_{1}^{4}+\left(\omega k_{1}+3 k_{2}^{2}+3 k_{3}^{2}\right)}{6 k_{1}^{2}}-k_{1}^{2} \mathrm{dc}^{2}\left(k_{1} x+k_{2} y+k_{3} z-\omega t\right) \\
& \pm k_{1}^{2} \sqrt{1-m^{2}} \mathrm{dc}\left(k_{1} x+k_{2} y+k_{3} z-\omega t\right) \operatorname{nc}\left(k_{1} x+k_{2} y+k_{3} z-\omega t\right) .
\end{aligned}
$$

Subcase 2.4. $c_{1}=-m^{2}, c_{2}=2, c_{3}=-\frac{1}{1-m^{2}}, c_{4}=\frac{1}{1-m^{2}}, c_{5}=-m^{2}, c_{6}=1$

Equations (4) and (6) have the solution $f(\xi)=\mathrm{dc} \xi, g(\xi)=\operatorname{sc} \xi \equiv \operatorname{sn} \xi / \mathrm{cn} \xi$. So the new periodic wave solution is

$$
\begin{aligned}
u= & \frac{\left(4 m^{2}+1\right) k_{1}^{4}+\left(\omega k_{1}+3 k_{2}^{2}+3 k_{3}^{2}\right)}{6 k_{1}^{2}}-k_{1}^{2} \mathrm{dc}^{2}\left(k_{1} x+k_{2} y+k_{3} z-\omega t\right) \\
& \pm k_{1}^{2} \sqrt{1-m^{2}} \mathrm{dc}\left(k_{1} x+k_{2} y+k_{3} z-\omega t\right) \operatorname{sc}\left(k_{1} x+k_{2} y+k_{3} z-\omega t\right) .
\end{aligned}
$$

As $m \rightarrow 0$, Eq. (24) degenerates to

$$
\begin{aligned}
u= & \frac{k_{1}^{4}+\left(\omega k_{1}+3 k_{2}^{2}+3 k_{3}^{2}\right)}{6 k_{1}^{2}}-k_{1}^{2} \sec ^{2}\left(k_{1} x+k_{2} y+k_{3} z-\omega t\right) \\
& \pm k_{1}^{2} \sec \left(k_{1} x+k_{2} y+k_{3} z-\omega t\right) \tan \left(k_{1} x+k_{2} y+k_{3} z-\omega t\right) .
\end{aligned}
$$

Case 3. $p=2 m^{2}-1, q=-2 m^{2}, r=1-m^{2}, c_{1}=m^{2}, c_{2}=-2 m^{2}$,

$$
c_{3}=1-m^{2}, c_{4}=m^{2}, c_{5}=m^{2}, c_{6}=-m^{2}
$$

In this case, we have $f(\xi)=\operatorname{cn} \xi, g(\xi)=\operatorname{dn} \xi$. Thus the periodic wave solution of Eq. (7) is

$$
\begin{aligned}
u= & -\frac{\left(5 m^{2}-1\right) k_{1}^{4}-\left(\omega k_{1}+3 k_{2}^{2}+3 k_{3}^{2}\right)}{6 k_{1}^{2}}+k_{1}^{2} m^{2} \operatorname{cn}^{2}\left(k_{1} x+k_{2} y+k_{3} z-\omega t\right) \\
& \pm k_{1}^{2} m \operatorname{cn}\left(k_{1} x+k_{2} y+k_{3} z-\omega t\right) \operatorname{dn}\left(k_{1} x+k_{2} y+k_{3} z-\omega t\right)
\end{aligned}
$$

Case 4. $p=2-m^{2}, q=-2\left(1-m^{2}\right), r=-1, c_{1}=1, c_{2}=-2\left(1-m^{2}\right)$,

$$
c_{3}=-\frac{1}{m^{2}}, c_{4}=\frac{1}{m^{2}}, c_{5}=1, c_{6}=-\left(1-m^{2}\right)
$$

The solution of Eqs. (4) and (6) reads $f(\xi)=\mathrm{nd} \xi, g(\xi)=\operatorname{sd} \xi$. So we get the periodic wave solution of Eq. (7)

$$
u=-\frac{\left(5-m^{2}\right) k_{1}^{4}-\left(\omega k_{1}+3 k_{2}^{2}+3 k_{3}^{2}\right)}{6 k_{1}^{2}}
$$




$$
\begin{aligned}
& +k_{1}^{2}\left(1-m^{2}\right) \text { nd }^{2}\left(k_{1} x+k_{2} y+k_{3} z-\omega t\right) \\
& \pm k_{1}^{2} m\left(1-m^{2}\right) \operatorname{nd}\left(k_{1} x+k_{2} y+k_{3} z-\omega t\right) \operatorname{sd}\left(k_{1} x+k_{2} y+k_{3} z-\omega t\right)
\end{aligned}
$$

Case 5. $p=2-m^{2}, q=2, r=1-m^{2}, c_{1}=1, c_{2}=2, c_{3}=1-m^{2}$,

$$
c_{4}=1, c_{5}=1, c_{6}=1
$$

From Eqs. (4) and (6) one obtains $f(\xi)=\operatorname{cs} \xi, g(\xi)=\mathrm{ds} \xi$. And the periodic wave solution of Eq. (7) reads

$$
\begin{aligned}
u= & -\frac{\left(5-m^{2}\right) k_{1}^{4}-\left(\omega k_{1}+3 k_{2}^{2}+3 k_{3}^{2}\right)}{6 k_{1}^{2}}-k_{1}^{2} \operatorname{cs}^{2}\left(k_{1} x+k_{2} y+k_{3} z-\omega t\right) \\
& \pm k_{1}^{2} \operatorname{cs}\left(k_{1} x+k_{2} y+k_{3} z-\omega t\right) \operatorname{ds}\left(k_{1} x+k_{2} y+k_{3} z-\omega t\right) .
\end{aligned}
$$

As $m \rightarrow 0$, Eq. (28) degenerates to Eq. (20).

Case 6. $p=2 m^{2}-1, q=2\left(1-m^{2}\right), r=-m^{2}, c_{1}=m^{2}, c_{2}=2\left(1-m^{2}\right)$,

$$
c_{3}=-1, c_{4}=1, c_{5}=m^{2}, c_{6}=1-m^{2}
$$

Equations (4) and (6) has the solution $f(\xi)=\mathrm{nc} \xi, g(\xi)=\mathrm{sc} \xi$. From Eq. (13) we get

$$
\begin{gathered}
u=-\frac{\left(5 m^{2}-1\right) k_{1}^{4}-\left(\omega k_{1}+3 k_{2}^{2}+3 k_{3}^{2}\right)}{6 k_{1}^{2}}-k_{1}^{2}\left(1-m^{2}\right) \mathrm{nc}^{2}\left(k_{1} x+k_{2} y+k_{3} z-\omega t\right) \\
\pm k_{1}^{2}\left(1-m^{2}\right) \mathrm{nc}\left(k_{1} x+k_{2} y+k_{3} z-\omega t\right) \operatorname{sc}\left(k_{1} x+k_{2} y+k_{3} z-\omega t\right) .
\end{gathered}
$$

As $m \rightarrow 0$, from Eq. (29) we get Eq. (25) again.

\section{Conclusion and discussion}

The exact travelling wave solutions to (3+1)-dimensional KP equation have been studied by means of the extended mapping method. Abundant periodic wave solutions in terms of Jacobi elliptic functions are obtained. Limit cases are studied and exact solitary wave solutions and trigonometric periodic wave solutions are got. Some of the solutions obtained in this paper develop singularity at a finite point, i.e. for any fixed $t=t_{0}$, there exists $x_{0}$ at which the solutions blow up. There is much current interest in the formation of so-called hot spots or blow up of solutions [13-16]. It appears that these singular solutions will model this physical phenomena. It can be easily seen that the method used in this paper is applicable to a large variety of nonlinear partial differential equations, as long as odd- and even-order derivative terms do not coexist in the equation under consideration.

\section{Acknowledgment}

This work is supported by the Postdoctoral Science Foundation of China. 


\section{References}

[1] Y.-Z. Peng Acta Phys. Pol. A 103, 417 (2003); 105, 417 (2004).

[2] Y.-Z. Peng, Phys. Lett. A 314, 401 (2003).

[3] Y.-Z. Peng, J. Phys. Soc. Jpn. 72, 1356 (2003).

[4] E. Yomba, Chaos, Solitons and Fractals 21, 209 (2004).

[5] V. Prasolov, Y. Solovyev, Elliptic Functions and Elliptic Integrals, American Mathematical Society, Providence 1997.

[6] M. Abramowitz, I.A. Stegun, Handbook of Mathematical Functions, Dover, New York 1972.

[7] R. Conte, M. Musette, Physica D 69, 1 (1993).

[8] M. Musette, R. Conte, J. Phys. A 27, 3895 (1994).

[9] M. Ablowitz, H. Segur, J. Fluid Mech. 92, 69 (1979).

[10] E.A. Kuznietsov, C.L. Musher, J. Exp. Theor. Phys. 63, 947 (1986).

[11] A. Senatorski, E. Infled, Phys. Rev. Lett. 77, 2855 (1986).

[12] T. Alagesan, A. Uthayakumar, K. Porsezian, Chaos, Solitons and Fractals 8, 893 (1997).

[13] N.A. Kudryashov, D. Zargaryan, J. Phys. A 29, 8067 (1996).

[14] C.J. Coleman, J. Aust. Math. Soc. Ser. B 33, 1 (1992).

[15] N.F. Smyth, J. Aust. Math. Soc. Ser. B 33, 403 (1992).

[16] P.A. Clarkson, E.L. Mansfield, Physica D 70, 250 (1993). 